\title{
Digital Image Segmentation Using SOM Network
}

\author{
Amera Istiqlal Badran \\ amera_istiqlal@uomosul.edu.iq \\ College of Computer Sciences and Mathematics \\ University of Mosul
}

\section{Received on:2009/9/28}

Accepted on:2010/4/11

\begin{abstract}
Image segmentation is one of the important stages in computer vision which is necessary for various applications such as robot control and identification of military targets, as will as image analysis of remote sensing applications.

In this paper the segmentation is implemented using k-means algorithm and minimum distance with and without SOM. Segmentation with SOM is done via many stages. In the first stage initialization and reading of image is done as well as type identification and normalization.

In the second stage the neural network SOM is implemented on the resultant image to extract its main colors. In the final stage image segmentation is done by clustering method using k-means algorithm with minimum distance. Segmentation is implemented by the following steps:-

* Image is segmented into two parts using two clusters centers.

* Calculation of a suggested quality factor to test segmentation quality for that number of clusters.

* Increment number of a clusters by one, calculate a new quality factor and compare it with the previous segmentation quality factor. Iterate this until the quality factor degrades and consider the previous classification as the right one.

* When fixing right clusters centers, a new image is created by substitution of image pixel with cluster center value that is nearest to the pixel value and then displaying and saving the final image.

Finally comparison is done between the four cases of results. It has been shown from result that the use of SOM with k-means \& Minimum distance algorithm in feasible, since it is depends on the variation of objects components of image.
\end{abstract}

Keywords: Image Segmentation, SOM Network. 


\title{
تقطيع الصور الرقمية الملونة باعتماد شبكة SOM
}

\author{
عامرة استقلال بدران \\ كلية علوم الحاسبات والرياضيات \\ جامعة الموصل
}

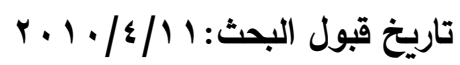

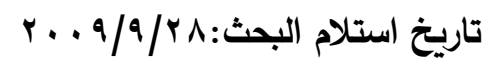

الملخص

إن تقطيع الصورة (Image Segmentation) هي إحدى أهم المراحل في الرؤية الحاسوبية

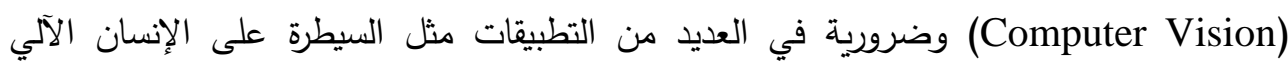

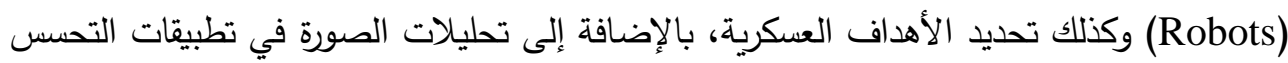

في هذا البحث تم تتفيذ عملية التقطيع باستخدام خوارزمية الـ M-means و Minimum

distance باعتماد شبكة الـ SOM وبدونها. إن إجراء عملية التقطيع باعتماد شبكة الـ SOM يتم فئت

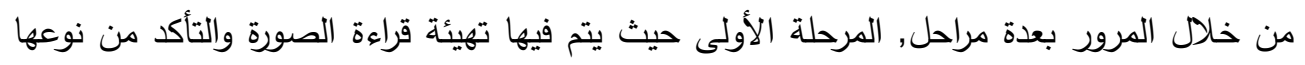

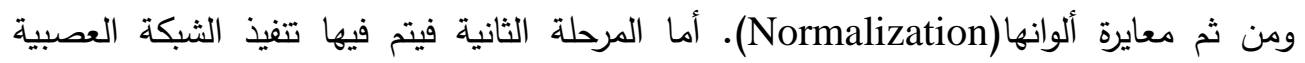
على الصورة الناتجة وذلك لاستخلاص الألوان الرئيسية من الصورة. أخيرا المرحلة الثالثة

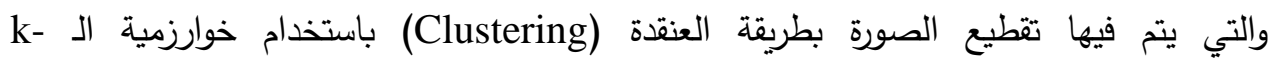

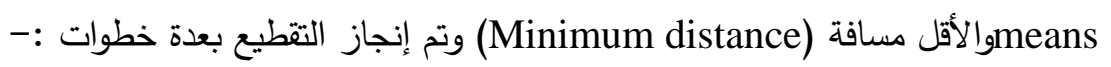
• • تقطيع الصورة إلى قسمين باعتبار مركزين عنقوديين. • حساب عامل جودة مقترحة لاختبار جودة التقطيع بهذا العدد من العناقيد.

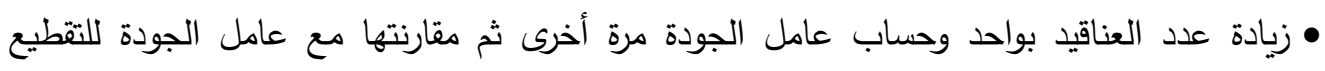
السابق، ونستمر بذلك طالما إن هناك تحسن بعامل الجودة، ثم نتوقف عند نقصانه ونعتبر التصنيف الذي سبق نقصان عامل الجودة هو التصنيف الصحيح.

• عند تثبيت مراكز عنقودية صحيحة يتم تكوين الصورة الجديدة وذلك بإعطاء نقاط الصورة (Pixels) قيمة المركز العنقودي الأقرب لقيمة النقطة وعرض الصورة النهائه تلئية وخزنها. 
وفي النهاية تم إجراء عملية مقارنة نتائج التقطيع من أربعة حالات الناتجة، فأظهرت النتائج مدى جدوى استخدام شبكة التظيم الذاتي(SOM) مع خوارزميتي الـ M-means و و من خلال اعتمادها على تفاوت في الكائنات المكونة للصورة. distance

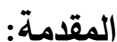

لقد وهب الله الإنسان القدرة على الإبصار وتمييز الأشياء مما يساعده على اتخاذ القرار، في

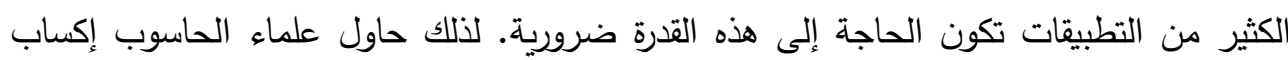
هذه القدرة للحاسوب ليتمكن من التعرف على مكونات الصورة، ويتم ذلك باستخدام مفاهيم الذكاء

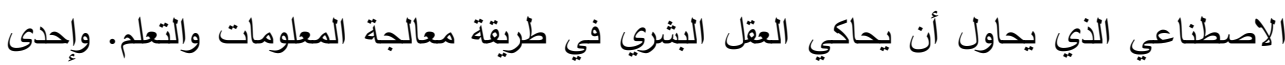
المراحل الضرورية للحصول على رؤية آلية هي تقطيع الصورة إلى مناطق تمثل مكونات الصورة. يهدف هذا البحث إلى الحصول على تقطيع للصورة مجهولة المكونات للحصول على مكونات الصورة بدون تدخل الإنسان، وهذا قد يكون مفيداً في الكثير من التطبيقات, منها في مجال التحكم

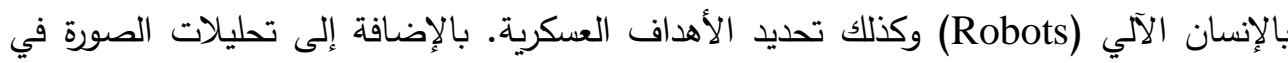
تطبيقات التحس النائي. وان الشبكة المستخدمة في هذا البحث هي شبكة التنظيم الذاتي

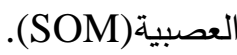

\section{شبكة التظيم الذاتي:}

شبكات التظيم الذاتي, والتي تدعى بشبكات (Kohonen). يوجد في هذه الثبكة عدد من

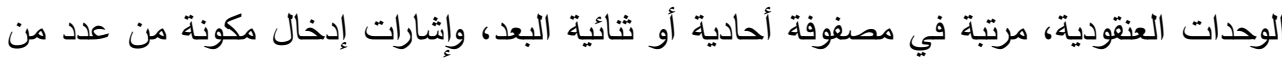
الوحدات. بمعنى آخر , يؤخذ في الحسبان الهيكل الهندسي بين الوحدات. إن كل إدخال يكون مرتبط

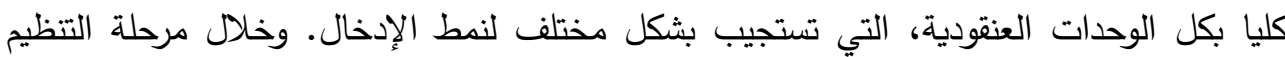
الذاتي فان الوحدة العنقودية التي متجه وزنها يجعل عينة الإدخال أكثر تقاربا من متجه التمثيل (أئي: أقل مسافة، لاحظ المعادلة (1)) فان هذه الوحدات ستُختار كوحدة فائزة، وفي كل خطوة من طور التدريب، فأن الوحدة العنقودية ذات الأوزان الأفضل مطابقة لنمط الإدخال تُنتخب كفائزة (winner)، هذه الوحدة الفائزة ومنطقة جوارها (الوحدات التي حولها) تُحدّث بطريقة بحيث أن النّان

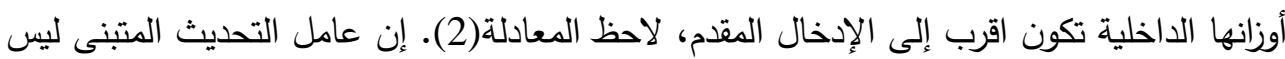
متساويا لجميع الخلايا. لكن يكون أقوى عند الوحدة الفائزة، ويتاقص عند الإدادئ الوحدات الأكثر بعداً 
$\left\|x(t)-W_{c}(t)\right\|=\min _{i}\left\{\left\|x(t)-W_{i}(t)\right\|\right\}$

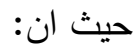

x : - متجه الادخال.

积

• $W_{i}$

t

تُعَّدث الأوزان مع الوقت، والعامل المكتسب يجب أن يخفض وأيضا الجوار يجب أن ينقص في

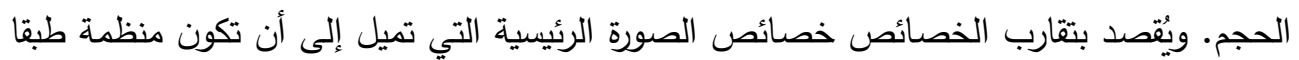

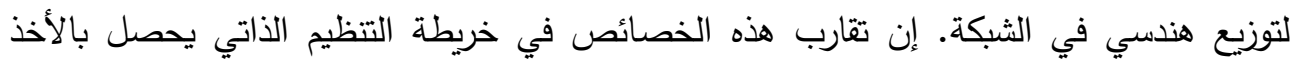
بنظر الاعتبار بعض التقييدات على العامل المكتسب أثناء تحديث الأوزان والتي تتم حسب فلب المعادلة التالية[2][6]:-

$W_{i}(t+1)=W_{i}(t)+\alpha(t)\left[x_{i}(t)-W_{i}(t)\right]$

$\forall i \in N c(t)$

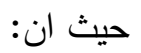

a

• مc Nc

الدالة المكتسبة المتبنية هي خطية، قيمها أعلى ما تكون في الوحدة الفائزة, وأقل ما تكون عند

حدود المنطقة الدائرية, لاحظ الثكل(1) [6].

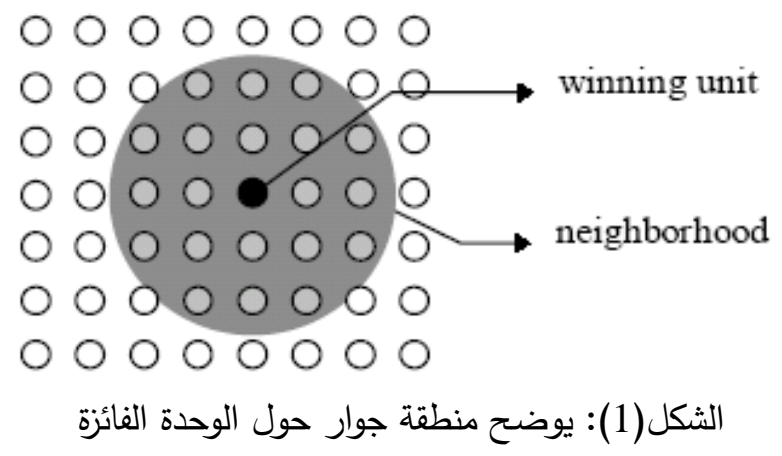

نصف قطر منطقة الجوار والدالة المكتسبة كلاهما تتاقصان في المقدار مع الوقت. هذا يتم بعاملي تخفيض، والتي تضرب في نصف القطر ومعاملات الدالة المكتسبة لكي تخفض قيمها. كلا 
القيمتين في المدى من \% 0 إلى 100\%. الـ 100\% يعني اضمحلال كلي, والـ 0\% يعني انه لا يوجد تخفيض. وعادة ما تكون قيمة صغيرة لمقدار التخفيض مطلوبة لانجاز تقارب فضاء خصائص الـ SOM [2]. يتم حساب المسافة الاقليدية للمناطق المتجاورة باعتماد المعادلة التالية $:[9][7]$ Dist $=\sqrt{\sum_{j=1}^{n}\left(x_{j}-W_{j}\right)^{2}}$

$$
\begin{aligned}
& \text { حيث ان: } \\
& \text { Dist } \\
& \text { n : عدد عناصر المصفوفة. }
\end{aligned}
$$

التقطيع (Segmentation):

يُعد التقطيع أحد أهم المراحل في أنظمة الرؤية الحاسوبية. وهو مهم في الكثير من تطبيقات الرؤية الحاسوبية ومعالجة الصور. والهدف من تقطيع الصورة هو إيجاد مناطق تمثل الأشياء (Objects)

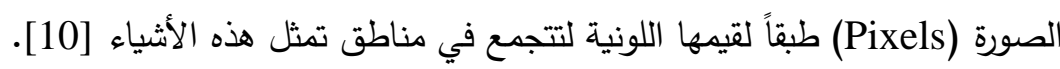

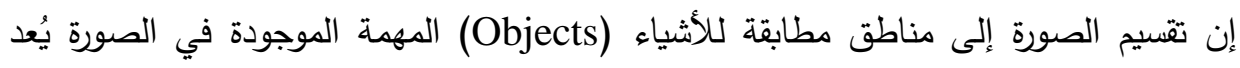

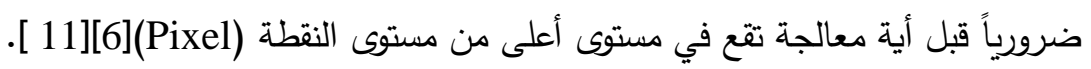

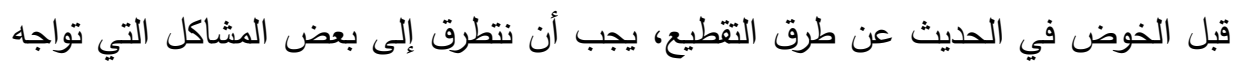

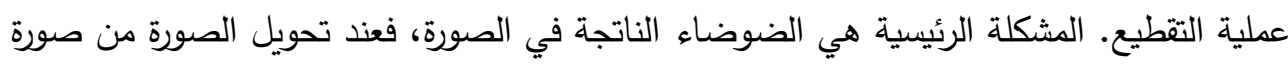

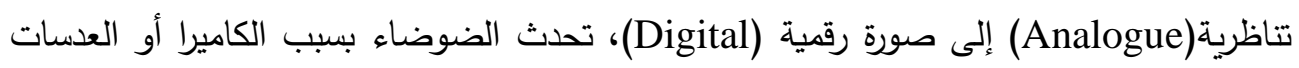
أو الإضاءة أو غير ذلك. ويمكن تقليل تأثيرها باستخدام طريقة من طرق التهيئة لما قبل الحبل المعالجة[10].

في هذا البحث تم التخلص من تأثير الإضاءة عن طريق معايرة ألوان الصورة

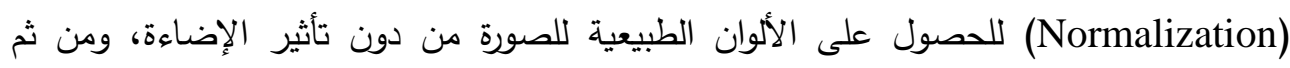

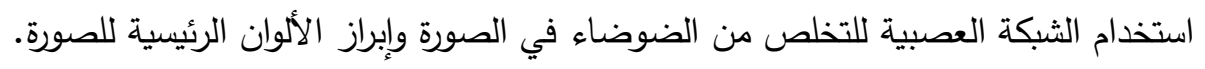

ولقد تم في هذا البحث استخدام طريقة العنقدة، وهي طريقة لتقطيع الصورة إلى مجموعات أو

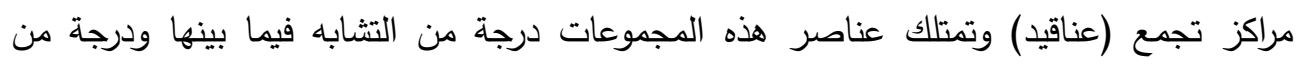

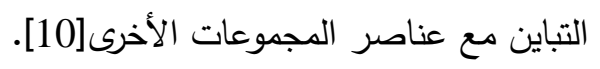


سنستخدم في هذا البحث التقطيع بطريقة العنقدة لأن التقطيع بطريقة اكتثاف الحواف

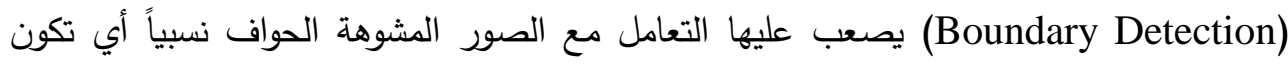
غير واضحة الحواف بينما التقطيع بطريقة نمو وتقلص المنطقة ( Region Growing and

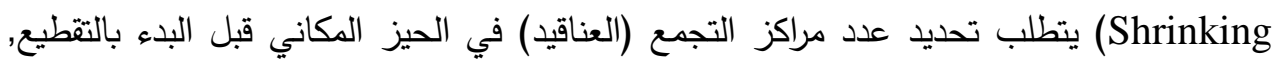
في حين أن طريقة العنقدة تستطيع التعامل مع الصور المشوهة الحواف والتي لا يمكن فيها تمييز

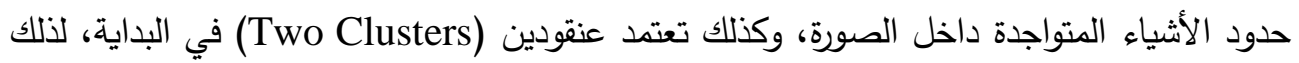
فضلنا استخدامها][3].

لقد ذكرنا سابقاً أن مفهوم طريقة العنقدة هو تقطيع الصورة إلى عدة مناطق تمثل عينات من

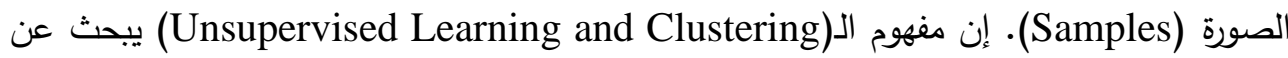
معلومات مستخلصة من عينات عشوائية، والطريقة العامة للعنقدة تُُّرف بعض مقاييس التثابه بين عنقودين إضافة إلى المقياس العام مثل مجموع مربع الأخطاء (Sum-squared error) أو مقدار

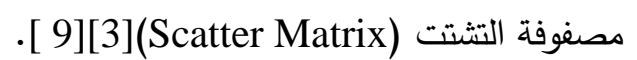
ويجب الإشارة هنا إلى أنه عندما نتعامل مع البيانات فإننا نتعامل مع عينات عشوائية من تلك الك البيانات, هذه العينات يمكن أن تثكل حشود من النقاط ( Pixels) في الفضاء المتعدد الأبعاد (Multidimensional space) (Mean and Covariance Matrix عينتين هما عينة المعدل وعينة مصفوفة التباين . وان عينة المعدل تحدد مركز الثقل في حشد معين من النقاط ومركز الثقل هو عبارة عن نقطة (Pixel) مفردة وهي أفضل تمثيل للبيانات ضمن مفهوم (أقل مجموع لمربع المسافات من فن فئن

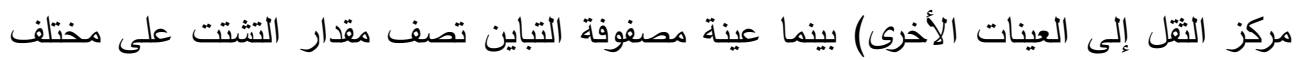
الاتجاهات. وهناك عدة خوارزميات للعنقدة منها [3]:

1- المسافة الأقل (Minimum Distance):

وهي إحدى الطرق المستخدمة في العنقدة حيث نختار نقطتين (Two Pixels) في البداية

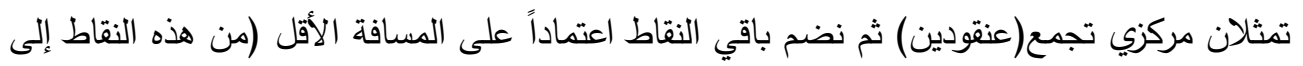

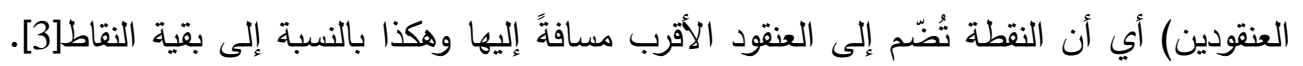
ويمكن تمثيل خوارزمية المسافة الأقل (Minimum Distance) بالخطوات التالية التالية:-

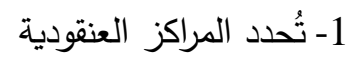

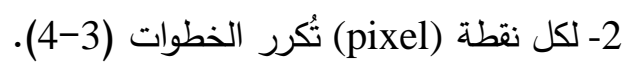

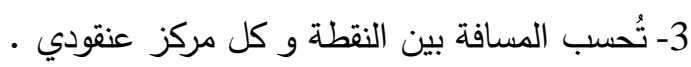




\section{4- تُضّم النقطة إلى العنقود الأقل مسافة . \\ 5- يُعطى لكل عنقود اللون الذي يمثل المركز العنقودي . \\ 2- k-means clustering العنقدة بعدة معدلات}

هي إحدى الطرق المستخدمة في العنقدة, ويتم فيها تحديد المراكز العنقودية وتنفيذ العنقدة باستخدام طريقة المسافة الأقل، ثم يحسب المعدل لكل عنقود ناتج وتعتبر كمراكز عنقودية جديدة، وتستمر هذه العملية إلى أن تثبت المراكز العنقودية[4][5]. وتمثل هذه الخوارزمية بالخطوات التالية

1- تقيئة المراكز العنقودية

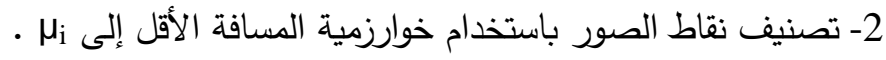

$$
\text { - }
$$

4- تكرر الخطوات (2-3) ما دامت بـ

$$
\text { . } \mu_{\mathrm{n}} \text {. . إرجاع }
$$

\section{مصفوفة التشتت (Scatter Matrix):}

ذكرنا سابقاً أن الطريقة العامة للعنقدة تكون عن طريق قياس التشابه بين عنقودين إضافة

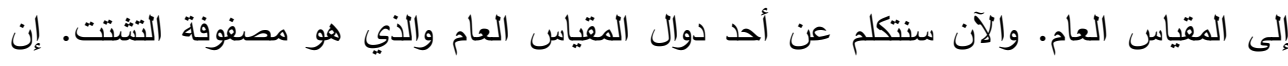
الغرض من استخدام هذه المقاييس هو لتحسين عمل العنقدة[3]. هناك نوعين من مصفوفات التشتت: - (الترصن

1. النوع الأول من مصفوفات التشتت يسىى التشتت الضمني، وهو عبارة عن مجموع تباعد كل عنصر عن مركز صنفه ونرمز لها بالرمز SW. ويتم حسابها كما في المعادلات التالية[6][6]

$S_{w}=\sum_{i=1}^{c} S_{i}$

$S_{i}=\sum_{x \in D_{i}}^{c}\left(x-m_{i}\right)\left(x-m_{i}\right)^{t}$

$m_{i}=\frac{1}{n_{i}} \underset{x \in D_{i}}{\sum x}$ 


$$
\begin{aligned}
& \operatorname{tr}\left[S_{W}\right]=\sum_{i=1}^{c} \sum_{x \in D_{i}}\left\|x-m_{i}\right\|^{2} \\
& \operatorname{tr}\left[S_{B}\right]=\sum_{i=1}^{c} n_{i}\left\|m_{i}-m\right\|^{2}
\end{aligned}
$$

حيث ان: Trace of a matrix : $\operatorname{tr}[]^{\text {. Tha }}$

عامل جودة التقطيع هو ناتج ضرب Trace لمصفوفة التشتت ضمن نفس العنقود في Trace لمصفوفة التشتت ما بين العناقيد، كما في المعادلة التالية[6].

$$
\beta=\operatorname{tr}\left[S_{W}\right] \operatorname{tr}\left[S_{B}\right]
$$

حيث ان:

- م

الطريقة المقترحة لعملية التقطيع:

: 1 التهيئة

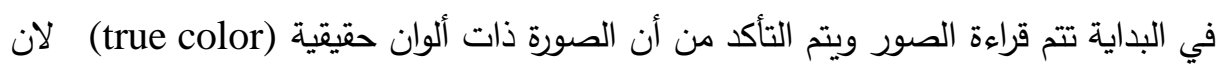

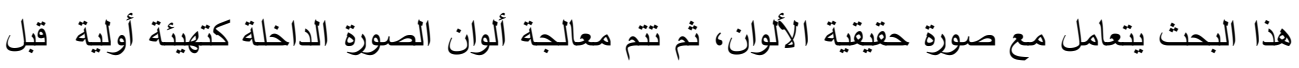
دخولها إلى الثبكة العصبية الاصطناعية. وذلك بمعايرة(Normalization) هذه الألوان باستخدام المعادلات التالية لتقليل تأثير الإضاءة على ألوان الصورة][1].

$$
\begin{aligned}
& r=\frac{R}{R+G+B} \\
& g=\frac{G}{R+G+B} \\
& b=\frac{B}{R+G+B}
\end{aligned}
$$


2- 2 تنفيذ خريطة التنظيم الذاتي:

بعد إتمام معايرة ألوان الصورة (Normalization) تدخل عينات من ألوان هذه الصورة إلى الى

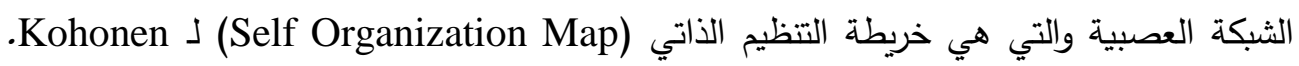
ويتم تنفيذ خوارزمية خريطة التظيم الذاتي كما يلي:

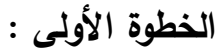

قبل البدء بتتفيذ الثبكة العصبية يجب تهيئة معاملات هذه الثبكة, وكما يلي: إن أوزان الثبكة تكون بأبعاد (32*32 ), كل منها مرتبط بثلاثة أوزان (تقابل مركبات ألوان الصورة الثلاثة RGB ). تتم تهيئة الأوزان بقيم عشوائية صغيرة ضمن المدى (1-0). بعد ذلك يتم تعيين نصف قطر منطقة الجوار التي يتم تحديث الأوزان ضمنها, وقد أعطينا القيمة (16) لنصف صنفيه قطر منطقة الجوار , وقد تم ذلك تجريبياً حيث أخُتبرت قيم أخرى مع تثبيت باقي المعاملات وحصلنا على أفضل النتائج عند هذه القيمة. القيم الأولية لدالة التحديث الخطية (م) عند الوحدة الفائزة هي (0.3)، وعند محيط دائرة منطقة الجوار (0.05)، وهذه القيم أيضاً جاءت نتيجة التجربة للحصول على أفضل النتائج .أما عاملا التخفيض لنصف قطر منطقة الجوار (0.001\%) وللدالة المكتسبة الخطية تم إعطائها (0.001\%)، وهنه وهذه القيم أيضاً جاءت نتيجة التجربة للحصول على أفضل أداء، وموازنة بين دقة النتائج وسرعة التتفيذ، مع إعطاء الأولوية لدقة النتائج[6]. الخطوة الثانية: طالما أن قيمة نصف القطر أكبر من أو تساوي (7) نفذ الخطوات من (3-7).

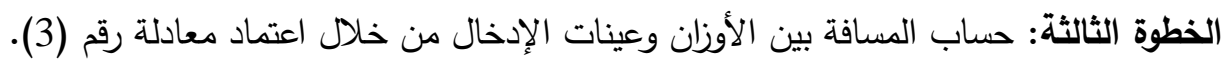
الخطوة الرابعة: إيجاد الوحدة الفائزة وتحديد موقعها من خلال اعتماد معادلة رقم (1). الخطوة الخامسة: تحديث الأوزان ضمن منطقة الجوار من خلال اعتماد معادلة رقم (2). الخطوة السادسة: تخفيض قيمة الدالة الخطية المكتسبة بمقدار عامل التخفيض(0.001). الخطوة السابعة: تقليل نصف قطر منطقة الجوار بمقدار عامل التخفيض المحدد في الخطوة رقم

بعد الانتهاء من تدريب الثبكة العصبية تكون الأوزان النهائية هي اخراجات من الثبكة

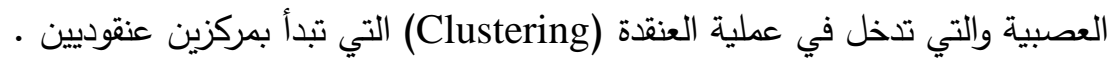


1) إن تحديد المراكز العنقودية قبل البدء بالعنقدة يكون باعتماد الخطوات التالية:

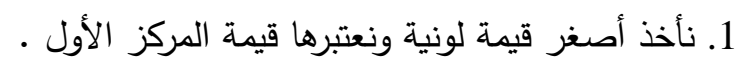

2. نأخذ أكبر قيمة لونية ونعتبرها قيمة المركز الأخير .

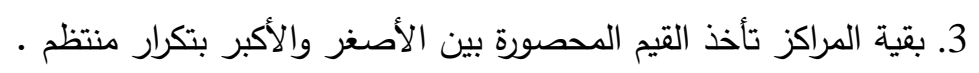

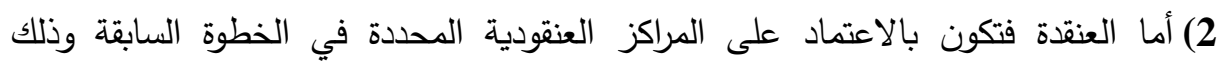

باعتماد احد الأسلوبين التاليين:

الأسلوب الأول/ المسافة الأقل وفيه يتم:

1. استلام المراكز العنقودية المحددة مسبقاً . المافل

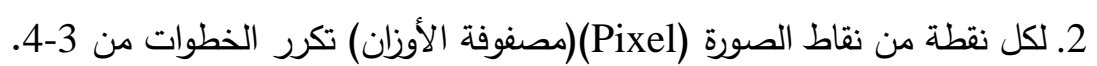

3. إيجاد الفرق بين قيمة النقطة (Pixel) الحالية والمراكز العنقودية .

4. تُضّم النقطة (pixel) إلى المركز العنقودي الأقل فرقاً (الأقل مسافة).

5. نعيد حساب المعدل للعناقيد (لتحديد لون العناقيد).

الأسلوب الثاني/k-means تم في هذا الأسلوب اعتماد خوارزمية k-means والذي يعتبر أسلوب

محدث عن أسلوب المسافة الأقل ويمكن تلخيصها بالخطوات التالية:

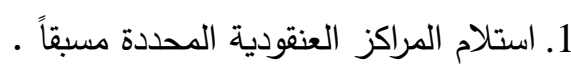

2. طالما أن المراكز العنقودية الحالية لا تساوي المراكز السابقة تكرر الخطوات من 3-

.6

3. لكل نقطة من نقاط الصورة (Pixel)(مصفوفة الأوزان) تكرر الخطوات من 4-5.

4. إيجاد الفرق بين قيمة النقطة (Pixel) الحالية والمراكز العنقودية .

5. تُضّم النقطة (pixel) إلى المركز العنقودي الأقل فرقاً (الأقرب مسافة).

6. نعيد حساب المعدل للعناقيد ( أي تكوين مراكز جديدة للعناقيد ).

3) كما يتم حساب عامل الجودة باعتماد الخطوات التالية:

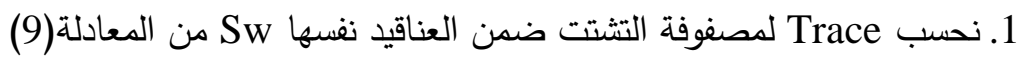

2. نحسب Trace لمصفوفة التثتت ما بين العناقيد SB من المعادلة (1)

3. يتم إيجاد المقياس العام (التشتت الكلي) من المعادلة (11).

4) بعدها يتم تكرار الخطوات من(1-3)طالما أن قيمة عامل الجودة الحالية أكبر من القيمة السابقة له. 
5) إذا كانت قيمة عامل الجودة الحالية أصغر من قيمة عامل الجودة السابقة فنوقف التكرار ونأخذ المراكز العنقودية السابقة والتي تعتبر هي المراكز العنقودية المطلوبة وتكون الصورة المقطعة الجديدة على أساس المراكز التالية:

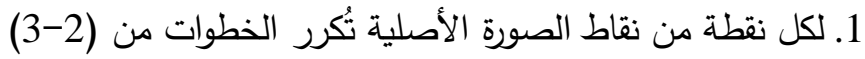
2. إيجاد الفرق بين قيمة النقطة والمراكز العنقودية . 3. إعطاء النقطة قيمة المركز العنقودي الأقل فرقاً (الأقرب مسافةً) .

إن المخطط الصندوقي لخوارزمية الطريقة المقترحة تم توضيحها في الثكل (2).

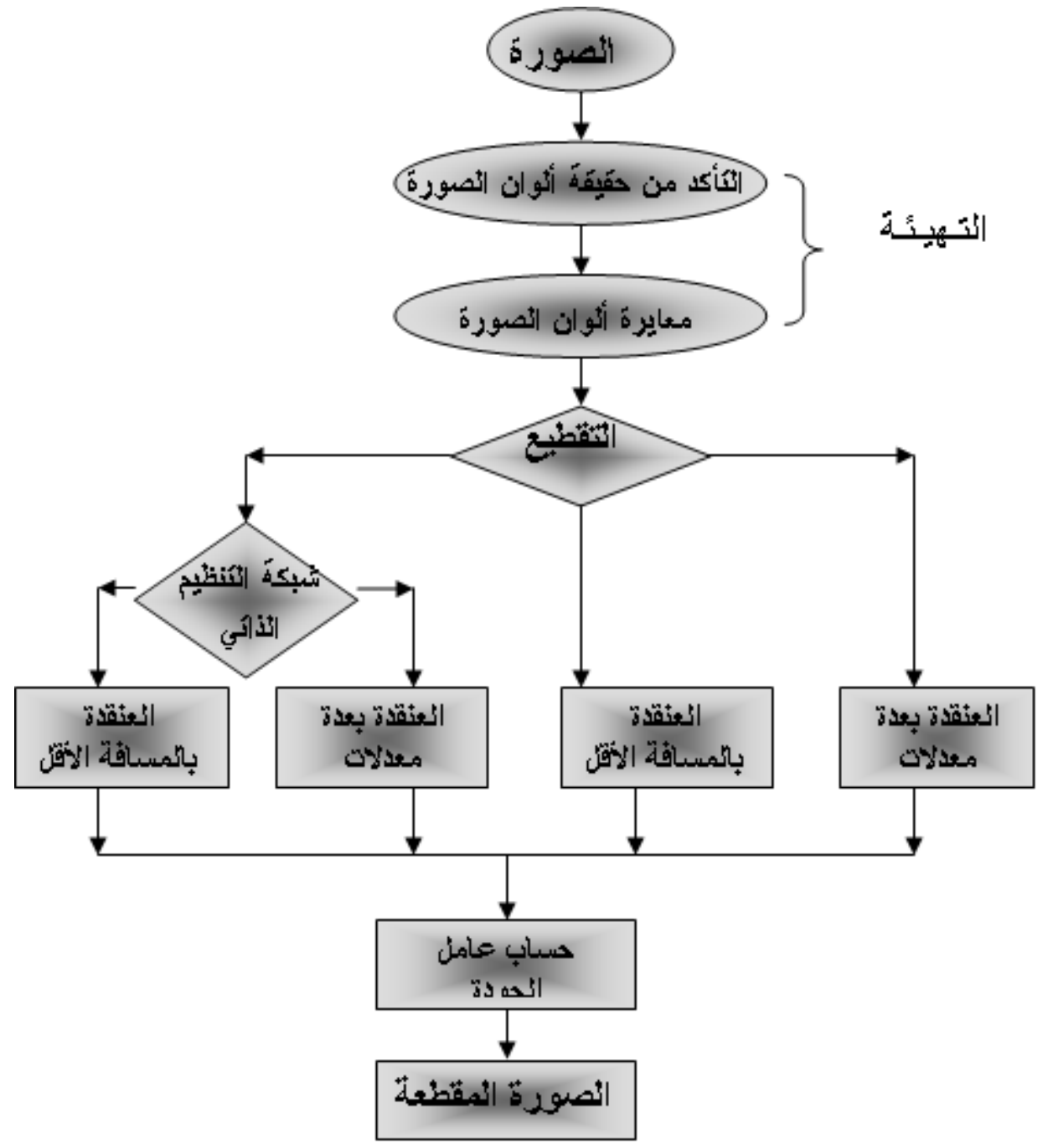

الثكل(2): يوضح المخطط الصندوقي للخوارزمية المقترحة 


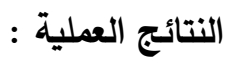

بعد إتمام البرنامج تم اختباره فأظهر كفاءة جيدة في عملية التقطيع مع سرعة جيدة في التنفيذ، والثكل (3) يبين مثال تطبيقي, حيث تم تتفيذ البرنامج على صورة جوية لأحد الأبنية في الجامعة, يمكن ملاحظة أنه تم التعرف على البنايات والظلال، وفصلها عن باقي المناطق. بعد الحصول

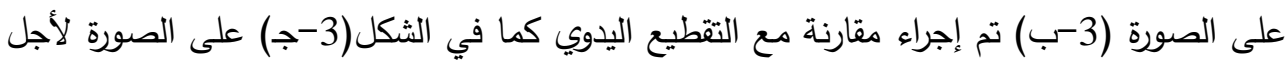
إيجاد المحتويات اللازمة للاستكمال الصورة.

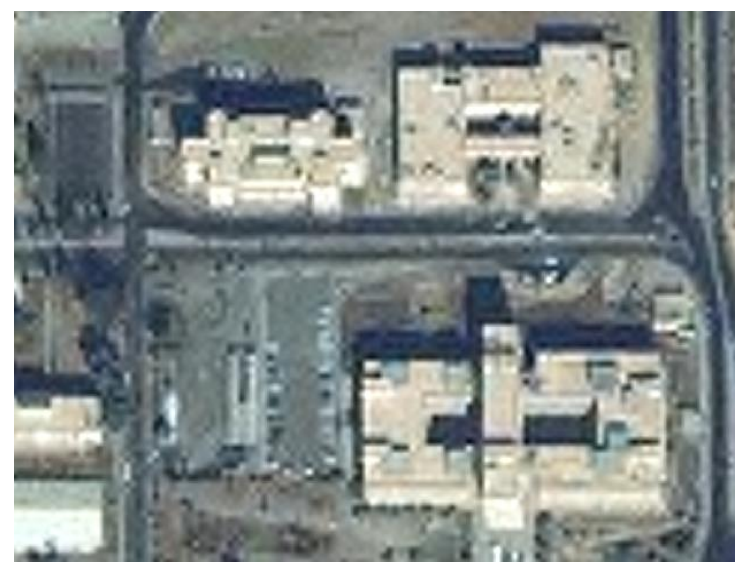

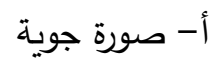

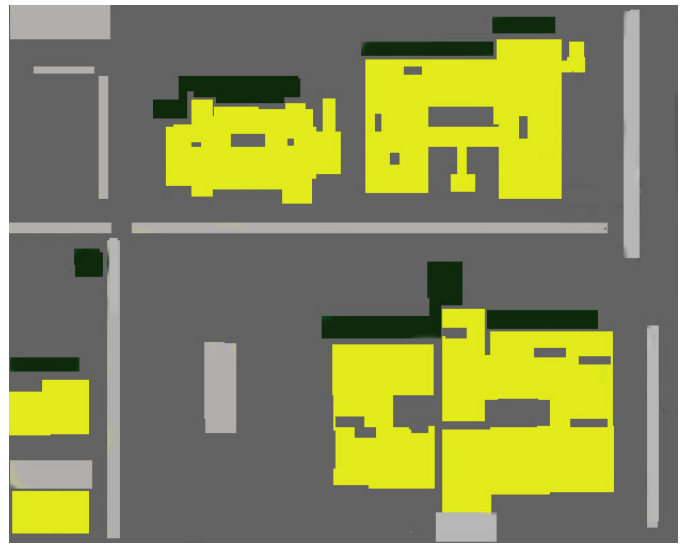

ج - صورة مقطعة تم تحسينها يدوياً

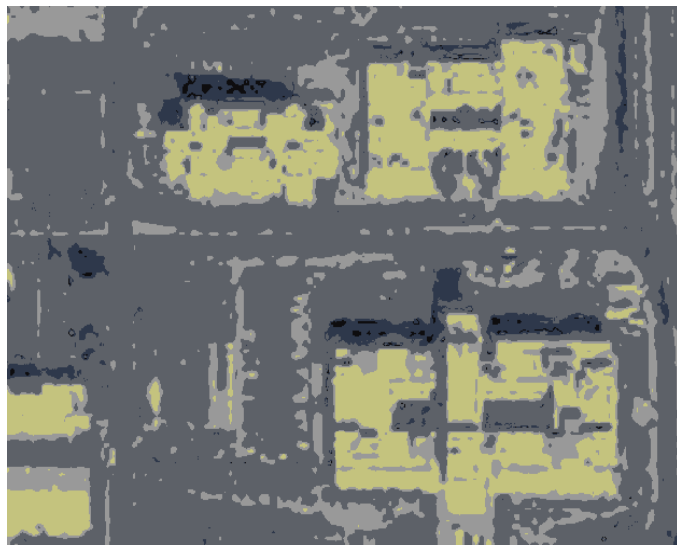

ب- - صورة مقطعة باستخدام الخوارزمية المقترحة

الثكل(3): يوضح مثال تطبيقي على عملية التقطيع 


\section{مقارنة بين خوارزميتي المسافة الأقل و العنقدة بعدة معدلات :}

لبيان مدى جدوى الطريقة المتبعة في هذا البحث من حيث خوارزمية العنقدة المستخدمة مع شبكة التظيم الذاتي (SOM)، تمت ألمقارنه بين النتائج في حالة استخدام خوارزمية المسافة الأقل

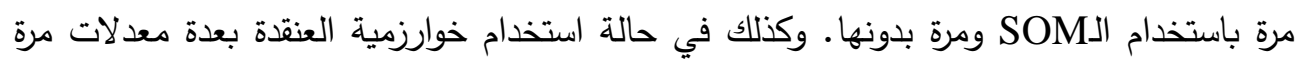

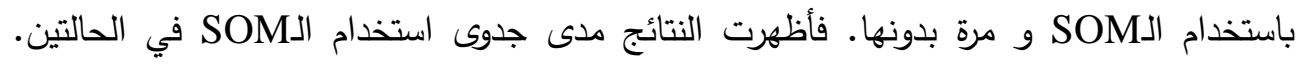

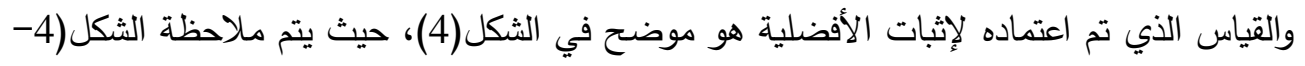

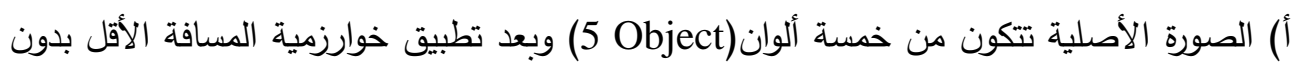

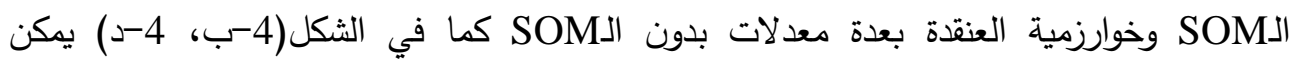

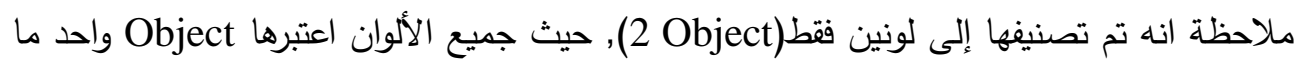
عدا اللون الأبيض والمقارب للأبيض اعتبرها Object آخر , أما عند تطبيق خوارزمية المسافة الأقل

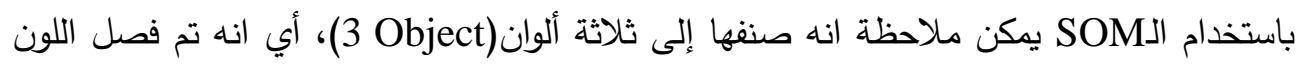
الأصفر عن اللون الأسود لأنه هناك فرق كبير بينهما واعتبر اللون الأصفر Object مستقل، كما انه يمكن ملاحظة ان هذا التصنيف أفضل من التصنيفين السابقين كما في الثكل(4-ج). أما عند تطبيق خوارزمية العنقدة بعدة معدلات باستخدام الـSOM يمكن ملاحظة انه صنفها أيضا إلى ثلاثة ألوان(3 Object) ولكنه فصل اللون الأحمر عن اللون الأسود وضم اللون الأحمر إلى الأصفر

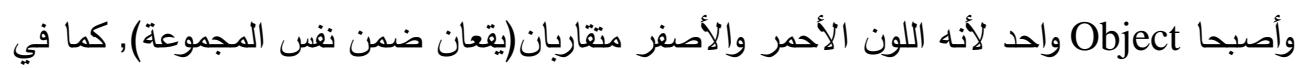
الثكل(4-هـ)، عليه يكون هذا التصنيف أفضل من التصنيفات السابقة. 


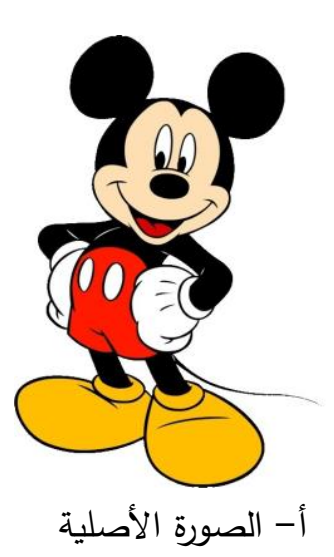

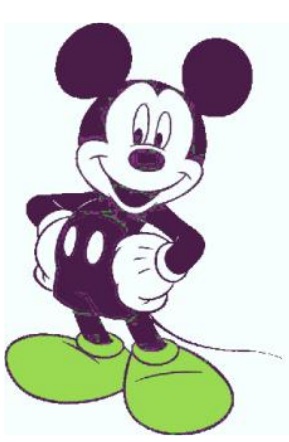

ج- خوارزمية المسافة الأقل باستخدام

الـ SOM

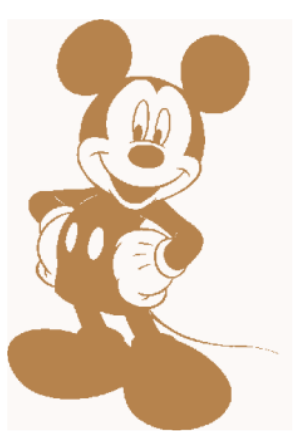

ب- خوارزمية المسافة الأقل بدون استخدام الـ SOM

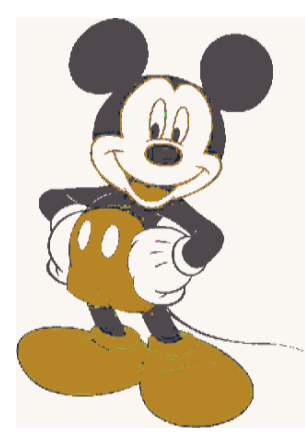

د - خوارزمية العنقدة بعدة معدلات

بدون استخدام الـ SOM

الثكل (4): يوضح مثال تطبيقي 
في هذا البحث تم كتابة كافة الدوال اللازمة لانجاز شبكة SOM مع البرمجيات الخاصة

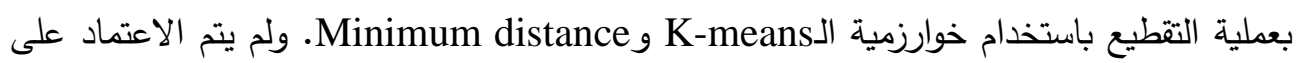

$$
\text { دوال جاهزه. }
$$

الاستنتاجات :

• تدريب شبكة التنظيم الذاتي على الصورة يعطي الألوان الرئيسية الموجودة في الصورة مما

$$
\text { يعطي نتائج أفضل في مرحلة العنقدة . }
$$

•الأوزان في شبكة الـ(SOM) عشوائية مما يؤدي إلى الابتعاد عن الحل المطلوب في

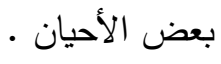

• استخدام خوارزمية الـ k-means في التصنيف يعطي نتائج أفضل (أقرب إلى الحل الصحيح) من خوارزمية الـ Minimum Distance وذلك بعد تكرار تصحيح المراكز العنقودية .

• عدد العينات المأخوذة من الصورة والتي تدخل على الثبكة العصبية ثابتة مما يجعل

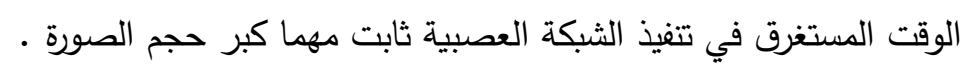

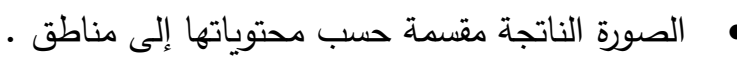

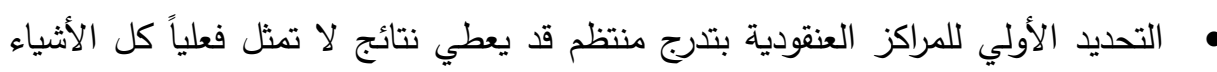

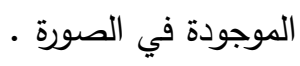




$$
\begin{aligned}
& \text { المصادر } \\
& \text { الطحان, نجم عبد الله عبد القادر سليمان,(2004), "كثف الوجه وتحديده بالصور الملونة", } \\
& \text { رسالة ماجستير , جامعة الموصل, كلية علوم الحاسبات والرياضيات, قسم علوم الحاسبات. } \\
& \text { عيسى, علام زكي, (2002), "الثبكات العصبية-البنية الهندسية-الخوارزميات- } \\
& \text { التطبيقات", شعاع للنشر والعلوم، سورية. }
\end{aligned}
$$

[3] Duda, Richard O. \& Hart, Peter E. \& Stork, David G., (2001), "Pattern Classification", Second Edition, Canada.

[4] E.S. Gopi, (2007), "Algorithm Collections for Digital Signal Processing Applications Using Matlab", National Institute of Technology, Tiruchi, India.

[5] F. van der Heijden, (2004)," Classification, Parameter Estimation and State Estimation", The Atrium, Southern Gate, Chichester, England.

[6] Luciano da Fontoura Costa \& Moreira, Jander , (1996), "Neuralbased color image segmentation and classification using selforganizing maps", Anais do Ix Sibgrapi.

[7] Marques de Sa, Joaquim P.,(2007),"Applied Statistics Using SPSS, Statistica, Matlab and R", Universidade do Porto, Fac. Engenharia.

[8] Martinez, Wendy L. \& Martinez, Angle R., (2005),"Exploratory Data Analysis with Matlab, ",London, New York. Washington, D.C.

[9] Martinez, Wendy L.\& Martinez, Angel R., (2002)," Computational Statistics Handbook with Matlab", Boca Raton London New York Washington, D.C..

[10] Scott E., Umbaugh, (1998), "Computer vision and image processing", A practical approach using CVIPtools" .

[11] Semmlow, John L., (2004), "Biosignal and Biomedical Image Processing" , Robert Wood Johnson Medical School, New Brunswick, Rutgers University, Piscataway, New Jersey, U.S.A.. 